ISSN (Online): 1402-9251 ISSN (Print): 1776-0852

f Journal Home Page: https://www.atlantis-press.com/journals/jnmp

\title{
Liouville Integrability of Conservative Peakons for a Modified CH equation
}

Xiangke Chang, Jacek Szmigielski

To cite this article: Xiangke Chang, Jacek Szmigielski (2017) Liouville Integrability of Conservative Peakons for a Modified CH equation, Journal of Nonlinear Mathematical Physics 24:4, 584-595, DOI: https://doi.org/10.1080/14029251.2017.1375693

To link to this article: https://doi.org/10.1080/14029251.2017.1375693

Published online: 04 January 2021 


\title{
Liouville Integrability of Conservative Peakons for a Modified CH equation
}

\author{
Xiangke Chang \\ LSEC, Institute of Computational Mathematics and Scientific Engineering Computing, AMSS, Chinese Academy of \\ Sciences, P.O.Box 2719, Beijing 100190, PR China \\ Department of Mathematics and Statistics, University of Saskatchewan, 106 Wiggins Road, Saskatoon, Saskatchewan, \\ S7N 5E6, Canada \\ changxk@lsec.cc.ac.cn \\ Jacek Szmigielski \\ Department of Mathematics and Statistics, University of Saskatchewan, 106 Wiggins Road, Saskatoon, Saskatchewan, \\ S7N 5E6, Canada \\ szmigiel@math.usask.ca
}

Received 4 May 2017

Accepted 17 June 2017

\begin{abstract}
The modified Camassa-Holm (also called FORQ) equation is one of numerous cousins of the Camassa-Holm equation possessing non-smoth solitons ( peakons) as special solutions. The peakon sector of solutions is not uniquely defined: in one peakon sector (dissipative ${ }^{\mathrm{a}}$ ) the Sobolev $H^{1}$ norm is not preserved, in the other sector (conservative), introduced in [2], the time evolution of peakons leaves the $H^{1}$ norm invariant. In this Letter, it is shown that the conservative peakon equations of the modified Camassa-Holm can be given an appropriate Poisson structure relative to which the equations are Hamiltonian and, in fact, Liouville integrable. The latter is proved directly by exploiting the inverse spectral techniques, especially asymptotic analysis of solutions, developed elsewhere [3].
\end{abstract}

Keywords: Conservative peakons; Liouville integrability.

2010 Mathematics Subject Classification: 35Q51, 37J35, 35Q53

\section{Introduction}

The partial differential equation with cubic nonlinearity

$$
\left.m_{t}+\left(\left(u^{2}-u_{x}^{2}\right) m\right)\right)_{x}=0, \quad m=u-u_{x x},
$$

is a modification of the Camassa-Holm equation $(\mathrm{CH})[1]$

$$
m_{t}+u m_{x}+2 u_{x} m=0, m=u-u_{x x},
$$

for the shallow water waves. The history of (1.1) is slightly convoluted: it appeared in the papers of Fokas [7], Fuchssteiner [8], Olver and Rosenau [15] and was, later, rediscovered by Qiao [16, 17]. Both equations have non-smooth solitons (called peakons) as solutions. In our recent Letter [2] we pointed out that (1.1) has in fact two meaningful types of peakon solutions: one type of peakon flows, based on the concept of weak solutions to conservation laws, proposed in [9], does

${ }^{\mathrm{a}}$ We are not implying that the Sobolev norm is decreasing. 
not preserve the $H^{1}$ norm $\|u\|_{H^{1}}$, the other type put forward in [2] does. For this reason we will refer to these two types of peakons as dissipative, conservative, respectively.

In this Letter we amplify the message of [2] by showing that in fact the conservative peakons form a Liouville integrable Hamiltonian system.

In the remainder of the introduction we argue why this is interesting, and certainly not automatic. Let us briefly recall the peakon setup for the $\mathrm{CH}$ equation (1.2), essentially in its original formulation [1]. The peakon Ansatz

$$
u=\sum_{j=1}^{n} p_{j}(t) e^{-\left|x-x_{j}(t)\right|}
$$

substituted into (1.2) results in the Hamiltonian system of equations:

$$
\dot{x}_{j}=\frac{\partial H}{\partial p_{j}}, \quad \dot{p}_{j}=-\frac{\partial H}{\partial x_{j}},
$$

with the Hamiltonian

$$
H=\frac{1}{2} \sum_{i, j=1}^{n} p_{i} p_{j} e^{-\left|x_{i}-x_{j}\right|}
$$

Its Liouville integrability was proven in [19] using the $R$-matrix formalism. We empasize that in the $\mathrm{CH}$ case the amplitudes $p_{j}$ and positions $x_{j}$ form conjugate pairs with respect to the canonical Poisson structure.

By contrast, in the case of equation (1.1), even though the peakon Ansatz looks superficially the same, namely $u=\sum_{j=1} m_{j}(t) e^{-\left|x-x_{j}(t)\right|}$, the candidates for momenta $m_{j}(t)$ are constant and one only gets a system of equations on the positions $x_{j}$ as shown in $[9,18]$ for dissipative peakons and in $[2,3]$ for conservative peakons. In either case it is not clear from the reduction point of view what part of the smooth structure survives the reduction to the peakon sector; for dissipative peakons, what constitutes the Hamiltonian in the smooth sector, namely the square of the $H^{1}$ norm, is not even a constant of motion in the peakon sector even though the norm is well defined.

To better explain out motivation let us consider the tempting possibility of reaching the conclusions of this paper by transforming (1.1) to one of the members of the AKNS hierarchy by using the reciprocal transformation:

$$
x=H(z, t), \quad d z=m d x-\left(\left(u_{x}^{2}-u^{2}\right) m\right) d t,
$$

discussed in [5, 6,11] and also [13] (in [11] (1.1) is called Qiao's equation). The success of such an approach is predicated on finding the change of variables $(x, t) \rightarrow(z=F(x, t), \tau=t)$ and the inverse $(z, t) \rightarrow(x=H(z, t), t=\tau)$. In the smooth sector the existence of $F(x, t)$ is guaranteed if (1.1) holds by elementary calculus of smooth differential forms (Poincare's Lemma). In our case, however, $m$ is a distribution and (1.1) would have to be taken as a distributional equation; what remains unclear is which one as there is more than one distributional analog of (1.1). Either way if $F$ existed it would have to be piecewise constant in $x$ since $F_{x}=m$ and $m$ is a discrete measure, making the transformation hard to interpret as a coordinate transformation. The situation with the existence of $H(z, t)$ is equally doubtful; were $H(z, t)$ to exist it would have to satisfy $H_{z}=\frac{1}{m}$, the inverse of the sum of the Dirac deltas does not seem to have a natural definition. This state of affairs is not without 
precedent both in the physics and mathematical literature. A case in point, in fact very pertinent to this discussion, is the 1-D Schrödinger equation

$$
-y_{x x}+u y=E y
$$

and the string equation:

$$
-v_{\xi \xi}=E m v
$$

The transformation (see [4]) $y=\sqrt[4]{m} v, \xi=\int_{0}^{x} \sqrt{m\left(\xi^{\prime}\right)} d \xi^{\prime}$ makes both equations equivalent, with $u$ and $m$ related by

$$
u=\frac{(\sqrt[4]{m})_{x x}}{m}
$$

provided $m$ is $C^{2}$ and $m>0$. When $m$ is a discrete measure, as it is in our case, the equivalence fails and these two equations are no longer equivalent, either physically, or mathematically.

In summary, we find it more compelling to study the peakon sector of (1.1) directly using well developed theory of distributions and then, if warranted, to investigate how, and if, to perform singular limits from the smooth sector of (1.1) (see interesting comments about this procedure in [13]).

For conservative peakons studied in this Letter, one is tempted to expect Liouville integrability based on the fact that the conservative peakon equations are derived from the compatibility conditions for a certain distributional Lax pair which was constructed in [3]. Indeed, we prove directly Liouville integrability by taking advantage of the inverse spectral solution formulas obtained in [3].

\section{Conservative peakons}

The equation (1.1) reduces to the conservative peakon sector [2] defined by the Ansatz

$$
u=\sum_{j=1}^{n} m_{j}(t) e^{-\left|x-x_{j}(t)\right|}
$$

and the multiplication rule

$$
u_{x}^{2} m \stackrel{\text { def }}{=}\left\langle u_{x}^{2}\right\rangle m
$$

where $\left\langle u_{x}^{2}\right\rangle m$ means that at a point $x_{j}$ of the singular support of $m=2 \sum_{j=1}^{n} m_{j} \delta_{x_{j}}$ one multiplies by the arithmetic average of the right and left limits of $u_{x}^{2}$ at $x_{j}$. The ensuing reduction is captured by the system of ODEs

$$
\dot{m}_{j}=0, \quad \dot{x}_{j}=u^{2}\left(x_{j}\right)-\left\langle u_{x}^{2}\right\rangle\left(x_{j}\right)
$$

on the weights $m_{j}$ of the measure $m$ and the points of singular support $x_{j}$. For later use we give two more explicit versions of the nontrivial part of the equations of motion: 


$$
\begin{aligned}
\dot{x}_{j}= & 2 \sum_{\substack{1 \leq k \leq n, k \neq j}} m_{j} m_{k} e^{-\left|x_{j}-x_{k}\right|}+ \\
& \sum_{k \neq j, i \neq j} m_{i} m_{k}\left(1-\operatorname{sgn}\left(x_{j}-x_{k}\right) \operatorname{sgn}\left(x_{j}-x_{i}\right)\right) e^{-\left|x_{j}-x_{k}\right|-\left|x_{j}-x_{i}\right|},
\end{aligned}
$$

and its more succinct form

$$
\dot{x}_{j}=2 \sum_{\substack{1 \leq k \leq n, k \neq j}} m_{j} m_{k} e^{-\left|x_{j}-x_{k}\right|}+4 \sum_{1 \leq i<j<k \leq n} m_{i} m_{k} e^{-\left|x_{i}-x_{k}\right|}, \quad 1 \leq j \leq n,
$$

valid when $x_{1}<x_{2}<\cdots<x_{n}$.

Inspired by papers by Hone and Wang [10,11], we have

Theorem 2.1. The equations (2.4) for the motion of $n$ peakons in the PDE (1.1) (with the condition (2.2) in place) are a Hamiltonian vector field:

$$
\dot{x}_{j}=\left\{x_{j}, h\right\}, \quad \dot{m}_{j}=\left\{m_{j}, h\right\},
$$

for the Hamiltonian

$$
h=\sum_{i, k=1}^{n} m_{i} m_{k} e^{-\left|x_{i}-x_{k}\right|}=\int u(\xi) m d \xi=\|u\|_{H^{1}}^{2}
$$

Here the Poisson bracket is given by

$$
\left\{x_{i}, x_{k}\right\}=\operatorname{sgn}\left(x_{i}-x_{k}\right), \quad\left\{m_{i}, m_{k}\right\}=\left\{m_{i}, x_{k}\right\}=0 .
$$

Proof. It is obvious that

$$
\left\{m_{j}, h\right\}=0
$$

under the above Poisson bracket, which leads to

$$
\dot{m}_{j}=0 \text {. }
$$

We proceed with the computation of $\left\{x_{j}, h\right\}$ :

$$
\begin{aligned}
\left\{x_{j}, h\right\} & =\left\{x_{j}, \sum_{i, k=1}^{n} m_{i} m_{k} e^{-\left|x_{i}-x_{k}\right|}\right\}=\left\{x_{j}, \sum_{i=1}^{n} m_{i}^{2}+2 \sum_{1 \leq i<k \leq n} m_{i} m_{k} e^{-\left|x_{i}-x_{k}\right|}\right\} \\
& =\left\{x_{j}, 2 \sum_{1 \leq i<k \leq n} m_{i} m_{k} e^{-\left|x_{i}-x_{k}\right|}\right\}=2 \sum_{1 \leq i<k \leq n} m_{i} m_{k}\left\{x_{j}, e^{-\left|x_{i}-x_{k}\right|}\right\} \\
& =2 \sum_{1 \leq i<k \leq n} m_{i} m_{k} \operatorname{sgn}\left(x_{k}-x_{i}\right) e^{-\left|x_{i}-x_{k}\right|}\left(\operatorname{sgn}\left(x_{j}-x_{i}\right)-\operatorname{sgn}\left(x_{j}-x_{k}\right)\right) \\
& =2 \sum_{1 \leq j=i<k \leq n}+2 \sum_{1 \leq i<k=j \leq n}+2 \sum_{1 \leq j<i<k \leq n}+2 \sum_{1 \leq i<k<j \leq n}+2 \sum_{1 \leq i<j<k \leq n},
\end{aligned}
$$


where we suppressed displaying the actual terms in the summation, concentrating on the ranges of summation instead. The first two summations give

$$
2 \sum_{1 \leq j=i<k \leq n}+2 \sum_{1 \leq i<k=j \leq n}=2 \sum_{\substack{1 \leq k \leq n, k \neq j}} m_{j} m_{k} e^{-\left|x_{j}-x_{k}\right|} .
$$

As for the last three three summations, we have

$$
\begin{aligned}
& 2 \sum_{1 \leq j<i<k \leq n}+2 \sum_{1 \leq i<k<j \leq n}+2 \sum_{1 \leq i<j<k \leq n} \\
& =\sum_{k \neq j, i \neq j} m_{i} m_{k} \operatorname{sgn}\left(x_{k}-x_{i}\right) e^{-\left|x_{i}-x_{k}\right|}\left(\operatorname{sgn}\left(x_{j}-x_{i}\right)-\operatorname{sgn}\left(x_{j}-x_{k}\right)\right) \\
& =\sum_{k \neq j, i \neq j} m_{i} m_{k}\left(1-\operatorname{sgn}\left(x_{j}-x_{k}\right) \operatorname{sgn}\left(x_{j}-x_{i}\right)\right) e^{-\left|x_{j}-x_{k}\right|-\left|x_{j}-x_{i}\right|},
\end{aligned}
$$

where the last equality is based on the facts that the corresponding equality holds for all the following cases

$$
\begin{aligned}
& x_{i}=x_{k}, \quad x_{i}=x_{j}, \quad x_{j}=x_{k}, \\
& x_{j}<x_{i}<x_{k}, \quad x_{i}<x_{j}<x_{k}, \quad x_{i}<x_{k}<x_{j}, \\
& x_{j}<x_{k}<x_{i}, \quad x_{k}<x_{j}<x_{i}, \quad x_{k}<x_{i}<x_{j} .
\end{aligned}
$$

Thus, we eventually get

$$
\left\{x_{j}, h\right\}=2 \sum_{\substack{1 \leq k \leq n, k \neq j}} m_{j} m_{k} e^{-\left|x_{j}-x_{k}\right|}+\sum_{k \neq j, i \neq j} m_{i} m_{k}\left(1-\operatorname{sgn}\left(x_{j}-x_{k}\right) \operatorname{sgn}\left(x_{j}-x_{i}\right)\right) e^{-\left|x_{j}-x_{k}\right|-\left|x_{j}-x_{i}\right|},
$$

which reproduces (2.4) and thus the proof is completed.

Remark 2.1. Observe that the Poisson bracket used above is a limiting case of a family of Poisson brackets discussed in [11]. In our case, if we restrict our considerations to the space of positions, then the Poisson bracket $\operatorname{sgn}\left(x_{i}-x_{k}\right)$ is up to a scale the skew-symmetric Green's function of the operator $D_{x}$.

For the remainder of this Letter we will focus on the following Poisson manifold $(M, \pi)$ defined with the help of the Poisson bracket (2.7).

Definition 2.1. Let

$$
M=\left\{x_{1}<x_{2}<\cdots<x_{n}\right\}
$$

and

$$
\pi(f, g)=\{f, g\}=\sum_{1 \leq i<j \leq n}\left\{x_{i}, x_{j}\right\} \frac{\partial f}{\partial x_{i}} \frac{\partial g}{\partial x_{j}}
$$

be defined for real valued functions $f, g$ on $M$. The Poisson manifold $M$ with the Poisson structure $\pi$ will be denoted $(M, \pi)$.

We will record the following result which trivially follows from (2.7) and the definition of rank of $\pi$ (see e.g. [12]).

Lemma 2.1. Let $n=2 K$ or $n=2 K+1$. Then the $\operatorname{rank}(\pi)=2 K$. 


\section{A manifold of conservative global peakons}

To simplify the presentation we will focus mostly on the case $n=2 K$. We will use $\mathbf{m}$ throughout this paper to denote the $n$-tuple of constant masses $m_{j}$. First we make a general observation about the nature of the vector field in (2.3): the vector field is discontinuous on the hyperplanes $x_{j}=x_{k}, j \neq k$. Let us then denote by $\mathscr{P}$ the set $\left\{\mathbf{m} ; x_{1}<x_{2}<\cdots<x_{2 K}\right\}$ of masses and positions where the vector field (see (2.4)) is Lipschitz, in fact smooth. The scattering map $\mathscr{S}$ used in [3] maps $\mathscr{P}$ to the set of admissible scattering data $\mathscr{R}=\{\mathbf{m} ; d \mu, c \geq 0\}$ consisting of the spectral measure $d \mu=\sum_{k=1}^{K} b_{k} \delta_{\zeta_{k}}, b_{k}>0$ and a constant $c$ which is 0 if $n=2 K$ and strictly positive if $n=2 K+1$. The problem is isospectral with the evolution of the spectral measure given by $d \mu(t)=e^{\frac{2 t}{\zeta}} d \mu(0)$. For an arbitrary choice of masses $m_{j}>0$ and initial positions $x_{j}(0)$ in (2.1) the flows are in general not global, since it can happen that $x_{j}(t)=x_{j+1}(t)$ for some $j$ at some finite time $t>0$. However, there exists a family of open subsets of $\mathscr{R}$ (see the Theorem below) for which the peakon flows are global, i.e. the solutions $x_{j}(t)$ exist for all $t \in \mathbf{R}$. This is crucial to our argument as we use the asymptotic behaviour of solutions to simplify the computations of Poisson brackets.

Theorem 3.1. [ [3], Case $n=2 K$ ] Given arbitrary spectral data

$$
\left\{b_{j}>0,0<\zeta_{1}<\zeta_{2}<\cdots<\zeta_{K}: 1 \leq j \leq K\right\}
$$

and denoting by $i^{\prime}=n+1-i$, suppose the masses $m_{k}$ satisfy

$$
\begin{array}{r}
\frac{\zeta_{K}^{\frac{k-1}{2}}}{\zeta_{1}^{\frac{k+1}{2}}}<m_{(k+1)^{\prime}} m_{k^{\prime}}, \quad \text { for all odd } k, \quad 1 \leq k \leq 2 K-1, \\
\frac{m_{(k+2)^{\prime}} m_{(k+1)^{\prime}}}{\left(1+m_{(k+1)^{\prime}}^{2} \zeta_{1}\right)\left(1+m_{(k+2)^{\prime}}^{2} \zeta_{1}\right)}<\frac{\zeta_{1}^{\frac{k+1}{2}}}{\zeta_{K}^{\frac{k-1}{2}}} \frac{2 \min _{j}\left(\zeta_{j+1}-\zeta_{j}\right)^{k-1}}{(k+1)\left(\zeta_{K}-\zeta_{1}\right)^{k+1}}, \\
\text { for all odd } k, \quad 1 \leq k \leq 2 K-3 .
\end{array}
$$

Then the positions obtained from inverse formulas in [3] are ordered $x_{1}<x_{2}<\cdots<x_{2 K}$ and the multipeakon solutions (2.1) exist for arbitrary $t \in \mathbf{R}$.

Remark 3.1. The odd case of $n=2 K+1$ is similar, although it requires a special care since in addition to $K$ eigenvalues $\zeta_{j}$ we also have an additional constant of motion, called $c$ in [3], which intuitively plays a role of an additional eigenvalue.

Finally, for computations, we will need the asymptotic form of global solutions. The theorem below is a slightly abbreviated form of the theorem presented in [3] .

Theorem 3.2. Suppose the masses $m_{j}$ satisfy the conditions of Theorem 3.1. Then the asymptotic position of a $k$-th (counting from the right) peakon as $t \rightarrow+\infty$ is given by

$$
\begin{array}{lr}
x_{k^{\prime}}=\frac{2 t}{\zeta_{\frac{k+1}{2}}}+C_{k}+\mathscr{O}\left(e^{-\alpha_{k} t}\right), & \text { for some positive } \alpha_{k}, C_{k} \in \mathbf{R}, \text { and odd } k, \\
x_{k^{\prime}}=\frac{2 t}{\zeta_{\frac{k}{2}}}+C_{k}+\mathscr{O}\left(e^{-\alpha_{k} t}\right), & \text { for some positive } \alpha_{k}, C_{k} \in \mathbf{R} \text { and even } k, \\
x_{k^{\prime}}=x_{(k+1)^{\prime}}=\ln m_{(k+1)^{\prime}} m_{k^{\prime}} \zeta_{\frac{k+1}{2}}+\mathscr{O}\left(e^{-\alpha_{k} t}\right), & \text { for some positive } \alpha_{k} \text { and odd } k .
\end{array}
$$


Likewise, as $t \rightarrow-\infty$, for convenience using the notation $l^{*}=K+1-l$, the asymptotic position of the $k$-th peakon is given by

$$
\begin{aligned}
& x_{k^{\prime}}=\frac{2 t}{\zeta_{\left(\frac{k+1}{2}\right)^{*}}}+D_{k}+\mathscr{O}\left(e^{\beta_{k} t}\right), \quad \text { for some positive } \beta_{k}, D_{k} \in \mathbf{R} \text { and odd } k, \\
& x_{k^{\prime}}=\frac{2 t}{\zeta_{\left(\frac{k}{2}\right)^{*}}}+D_{k}+\mathscr{O}\left(e^{\beta_{k^{t}}}\right), \quad \text { for some positive } \beta_{k}, D_{k} \in \mathbf{R} \text { and even } k, \\
& x_{k^{\prime}}-x_{(k+1)^{\prime}}=\ln m_{(k+1)^{\prime}} m_{k^{\prime}} \zeta_{\left(\frac{k+1}{2}\right)^{*}}+\mathscr{O}\left(e^{\beta_{k} t}\right), \quad \text { for some positive } \beta_{k} \text { and odd } k .
\end{aligned}
$$

Remark 3.2. We emphasize that in spite of notational complexity the most essential features of the theorem can be stated simply: asymptotically, particles form bound states consisting of adjacent particles sharing asymptotic velocities $\frac{2}{\zeta_{j}}, j=1, \ldots, K$ and moving in pairs, each pair moving as if it were a free particle. This picture persists if $n=2 K+1$ with the only exception that for large positive times the first particle, counting from the left, comes to a stop, while the remaining particles pair up the same way they do for $n=2 K$. Likewise, for large negative times, the first particle, counting from the right, comes to a stop, while the rest of particles pair up.

We end this subsection with the corollary which will be used in the proof of Liouville integrability of the peakon system (2.5). We will state this lemma in a slightly informal way by emphasizing the role of asymptotic pairs discussed in Remark 3.2.

Corollary 3.1. Let $n=2 K$ then asymptotically pairs of peakons scatter, that is the distances between particles from distinct pairs diverge to $\infty$. If $n=2 K+1$ and one counts the first particle, counting from the left, as a "pair" then asymptotically, as $t \rightarrow \infty$, the pairs scatter. Likewise, if one counts the first particle, counting from the right, as a " pair" then asymptotically, as $t \rightarrow-\infty$, the pairs scatter.

\subsection{Liouville integrability}

We need to introduce a bit of notation to facilitate the presentation of formulas and subsequent computations. Most of the computations in this Letter involve a choice of $j$-element index sets $I$ and $J$ from the set $[k]=\{1,2, \ldots, k\}$. We will use the notation $\left(\begin{array}{c}{[k]} \\ j\end{array}\right)$ for the set of all $j$-element subsets of $[k]$, listed in increasing order; for example $I \in\left(\begin{array}{c}{[k]} \\ j\end{array}\right)$ means that $I=\left\{i_{1}, i_{2}, \ldots, i_{j}\right\}$ for some increasing sequence $i_{1}<i_{2}<\cdots<i_{j} \leq k$. Furthermore, given the multi-index $I$ we will abbreviate $g_{I}=g_{i_{1}} g_{i_{2}} \ldots g_{i_{j}}$ etc.

Definition 3.1. Let $I, J \in\left(\begin{array}{c}{[k]} \\ j\end{array}\right)$, or $I \in\left(\begin{array}{c}{[k]} \\ j+1\end{array}\right), J \in\left(\begin{array}{c}{[k]} \\ j\end{array}\right)$.

Then $I, J$ are said to be interlacing if

$$
i_{1}<j_{1}<i_{2}<j_{2}<\cdots<i_{j}<j_{j}
$$

or,

$$
i_{1}<j_{1}<i_{2}<j_{2}<\cdots<i_{j}<j_{j}<i_{j+1},
$$

in the latter case. We abbreviate this condition as $I<J$ in either case, and, furthermore, use the same notation, that is $I<J$, for $I \in\left(\begin{array}{c}k] \\ 1\end{array}\right), J \in\left(\begin{array}{c}{[k]} \\ 0\end{array}\right)$. 
(1) Case $n=2 K$.

It was shown in [3] that the quantities

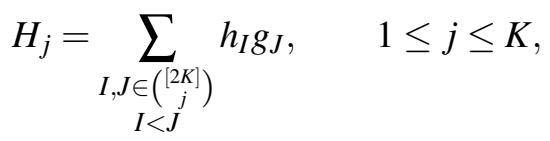

with $h_{i}=m_{i} e^{x_{i}}, g_{i}=m_{i} e^{-x_{i}}$, form a set of $K$ constants of motion for the system (2.5) in the even case $n=2 K$. In particular the Hamiltonian $h$ in Theorem 2.1 satisfies

$$
h=2 H_{1}+\sum_{i=1}^{n} m_{i}^{2}
$$

We need the following computational result.

Lemma 3.1. Consider the Poisson bracket given in Definition 2.1. Then

$$
\left\{\prod_{p \in I,|I|=i} m_{2 p-1} m_{2 p} e^{x_{2 p-1}-x_{2 p}}, \prod_{q \in J,|J|=j} m_{2 q-1} m_{2 q} e^{x_{2 q-1}-x_{2 q}}\right\}=0 .
$$

Proof. Let us denote

$$
\prod_{p \in I,|I|=i} m_{2 p-1} m_{2 p} e^{x_{2 p-1}-x_{2 p}}=F, \quad \prod_{q \in J,|J|=j} m_{2 q-1} m_{2 q} e^{x_{2 q-1}-x_{2 q}}=G .
$$

Then, by elementary properties of exponentials and basic properties of Poisson brackets, we have

$$
\{F, G\}=F G \sum_{p \in I, q \in J}\left\{x_{2 p-1}-x_{2 p}, x_{2 q-1}-x_{2 q}\right\} .
$$

However,

$$
\begin{aligned}
\left\{x_{2 p-1}-x_{2 p}, x_{2 q-1}-x_{2 q}\right\} & =\left\{x_{2 p-1}, x_{2 q-1}\right\}-\left\{x_{2 p-1}, x_{2 q}\right\}-\left\{x_{2 p}, x_{2 q-1}\right\}+\left\{x_{2 p}, x_{2 q}\right\} \\
& =\operatorname{sgn}(p-q)-\operatorname{sgn}(p-q)-\operatorname{sgn}(p-q)+\operatorname{sgn}(p-q)=0 .
\end{aligned}
$$

Thus the claim is proved.

Theorem 3.3. The Hamiltonians $H_{1}, \cdots, H_{K}$ Poisson commute.

Proof. The idea of the proof goes back at least to the work of J. Moser on the finite Toda lattice [14]. In the nutshell it amounts to the following observation: the Poisson bracket $\left\{H_{i}, H_{j}\right\}\left(\mathbf{x}^{0}\right)$ of two conserved quantities $H_{i}, H_{j}$ is also conserved and thus instead of the fixed point $\mathbf{x}^{0}$ it can be evaluated on the orbit $\mathbf{x}(t)$ going through $\mathbf{x}^{0}$, in particular in the asymptotic region $t \rightarrow \pm \infty$, if such is accessible. In our case both asymptotic regions can be used but for the sake of argument we will focus on $t \rightarrow-\infty$. 
Note that, in view of (3.4), and for large negative times, the only contributing terms from individual $H_{i}$ s are

$$
\sum_{I \in\left(\begin{array}{c}
{[K]} \\
i
\end{array}\right)} \prod_{p \in I} m_{2 p-1} m_{2 p} e^{x_{2 p-1}-x_{2 p}}
$$

hence

$$
\begin{aligned}
& \left\{H_{i}, H_{j}\right\}\left(\mathbf{x}^{0}\right)=\left\{H_{i}, H_{j}\right\}(\mathbf{x}(t))=\lim _{t \rightarrow-\infty}\left\{H_{i}, H_{j}\right\}(\mathbf{x}(t)) \\
= & \lim _{t \rightarrow-\infty}\left\{\sum_{I \in\left(\begin{array}{c}
{[K]} \\
i
\end{array}\right)} \prod_{p \in I} m_{2 p-1} m_{2 p} e^{x_{2 p-1}-x_{2 p}}, \sum_{J \in\left(\begin{array}{c}
{[K]} \\
j
\end{array}\right)} \prod_{q \in J} m_{2 q-1} m_{2 q} e^{x_{2 q-1}-x_{2 q}}\right\}(\mathbf{x}(t)) \\
= & \lim _{t \rightarrow-\infty} \sum_{I \in\left(\begin{array}{c}
{[K]} \\
i
\end{array}\right)} \sum_{J \in\left(\begin{array}{c}
{[K]} \\
j
\end{array}\right)}\left\{\prod_{p \in I} m_{2 p-1} m_{2 p} e^{x_{2 p-1}-x_{2 p}}, \prod_{q \in J} m_{2 q-1} m_{2 q} e^{x_{2 q-1}-x_{2 q}}\right\}(\mathbf{x}(t)) .
\end{aligned}
$$

By using Lemma 3.1 the final conclusion follows.

(2) Case $n=2 K+1$.

Again, following [3],

$$
H_{j}=\sum_{\substack{I, J \in\left(\begin{array}{c}
{[2 K+1] \\
I<J}
\end{array}\right) \\
j}} h_{I} g_{J}, \quad 1 \leq j \leq K,
$$

with $h_{i}=m_{i} e^{x_{i}}, \quad g_{i}=m_{i} e^{-x_{i}}$, are constants of motion for the system (2.5) in the odd case.

In the odd case, there is an extra constant of motion, which can be computed from the value of the Weyl function at $\infty$ in the spectral variable. The computation is routine and produces (see Section III of [3] for details regarding the Weyl function)

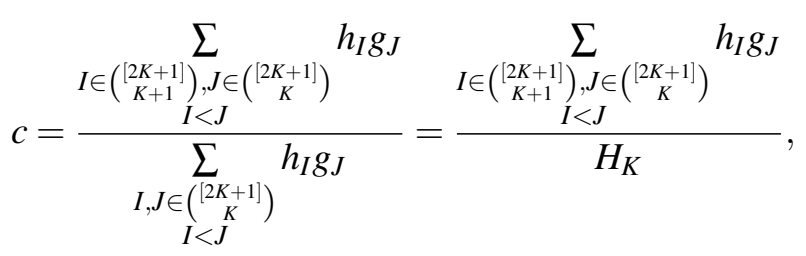

which, in turn, gives an extra constant of motion

$$
H_{c}=\sum_{\substack{I \in\left(\begin{array}{c}
{[2 K+1] \\
K+1}
\end{array}\right), J \in\left(\begin{array}{c}
{[2 K+1] \\
K}
\end{array}\right) \\
I<J}} h_{I} g_{J}=\prod_{j=1}^{2 K+1} m_{j} e^{(-1)^{j+1} x_{j}}
$$

so that $\left\{H_{1}, H_{2}, \cdots, H_{k}, H_{c}\right\}$ form a set of $K+1$ constants of motion for the system (2.5) in this case.

Theorem 3.4. The Hamiltonians $H_{1}, \cdots, H_{K}, H_{c}$ Poisson commute . 
Proof. Again, asymptotically for large negative times, the $H_{j}$ s simplify to

$$
\sum_{I \in\left(\begin{array}{c}
(K] \\
j
\end{array}\right)} \prod_{j \in I} m_{2 j-1} m_{2 j} e^{x_{2 j-1}-x_{2 j}}
$$

Then the argument from the previous theorem applies verbatim, proving that

$$
\left\{H_{i}, H_{j}\right\}=0
$$

holds.

We turn now to proving that the $H_{j}$ s and the $H_{c}$ are in involution. A direct computation gives:

$$
\begin{aligned}
& \left\{H_{j}, H_{c}\right\}\left(\mathbf{x}^{0}\right)=\left\{H_{j}, H_{c}\right\}(\mathbf{x}(t))=\lim _{t \rightarrow-\infty}\left\{H_{j}, H_{c}\right\}(\mathbf{x}(t)) \\
= & \lim _{t \rightarrow-\infty}\left\{\sum_{J \in\left(\begin{array}{c}
{[K]} \\
j
\end{array}\right)} \prod_{p \in J} m_{2 p-1} m_{2 p} e^{x_{2 p-1}-x_{2 p}}, \prod_{q=1}^{2 K+1} m_{q} e^{(-1)^{q+1} x_{q}}\right\}(\mathbf{x}(t)) \\
= & \lim _{t \rightarrow-\infty} \sum_{J \in\left(\begin{array}{c}
{[K]} \\
j
\end{array}\right)}\left\{\prod_{p \in J} m_{2 p-1} m_{2 p} e^{x_{2 p-1}-x_{2 p}}, \prod_{q=1}^{2 K+1} m_{q} e^{(-1)^{q+1} x_{q}}\right\}(\mathbf{x}(t)) \\
= & \lim _{t \rightarrow-\infty} \sum_{J \in\left(\begin{array}{c}
{[K]} \\
j
\end{array}\right)} \prod_{p \in J} m_{2 p-1} m_{2 p} e^{x_{2 p-1}-x_{2 p}} \prod_{q=1}^{2 K+1} m_{q} e^{(-1)^{q+1} x_{q}} \\
\cdot & \sum_{p \in J}\left(\sum_{q=1}^{K}\left\{x_{2 p-1}-x_{2 p}, x_{2 q-1}-x_{2 q}\right\}+\left\{x_{2 p-1}-x_{2 p}, x_{2 K+1}\right\}\right)(\mathbf{x}(t))
\end{aligned}
$$

We have shown in the course of proving Lemma 3.1 that

$$
\left\{x_{2 p-1}-x_{2 p}, x_{2 q-1}-x_{2 q}\right\}=0 .
$$

Furthermore,

$$
\left\{x_{2 p-1}-x_{2 p}, x_{2 K+1}\right\}=\operatorname{sgn}\left(x_{2 p-1}-x_{2 K+1}\right)-\operatorname{sgn}\left(x_{2 p}-x_{2 K+1}\right)=0
$$

holds. Thus, indeed

$$
\left\{H_{j}, H_{c}\right\}\left(\mathbf{x}^{0}\right)=0
$$

and the proof is completed.

Theorem 3.5. The conservative peakon system given by (2.5) is Liouville integrable.

Proof. To prove Liouville integrability of a Hamiltonian system defined on an $n$-dimensional Poisson manifold $(M, \pi)$ we need to show that the integrals of motion are functionally independent, they commute and the number of them, say $s$, satisfies $\frac{1}{2} \operatorname{rank}(\pi)+s=n$ [12]. In the case at hand, by Lemma 2.1, $\operatorname{rank}(\pi)=K$ and since we have $s=K$ commuting Hamiltonians for $n=2 K$ and $s=K+1$ commuting Hamiltonians for $n=2 K+1$, the relation $\frac{1}{2} \operatorname{rank}(\pi)+s=n$ holds in both cases. To prove the functional independence we proceed as follows. We need to prove that 
$\Omega=d H_{1} \wedge d H_{2} \wedge \cdots \wedge d H_{S}$ is non-zero on a dense subset of $M$. Since $H_{i}$ s are rational functions of $\xi_{l}=e^{x_{l}}, l=1, \ldots, n$, so is $\Omega$. It is thus sufficient to show that $\Omega$ is not identically zero. In the asymptotic regions used in the proofs of Theorem 3.3 and $3.4 H_{j}$ s are asymptotically polynomials in the variables $e^{x_{2 p-1}-x_{2 p}}$ of degree $j$ (see e.g. (3.5)) and, conseqently, $\Omega$ is not identically zero in that region, which concludes the proof.

We finish this section by observing that it is elementary to identify the Darboux coordinates in the case $n=2 K$. Indeed, suppose we introduce coordinates

$$
\left\{p_{k}, q_{k}, k=1,2, \ldots, K\right\},
$$

by defining

$$
p_{k}=x_{2 k}-x_{2 k-1}, \quad q_{k}=\sum_{j=1}^{k}\left(x_{2 j-1}-x_{2 j-2}\right)
$$

with the convention that $x_{0}=0$. Then by a direct computation we obtain

$$
\left\{p_{k}, q_{m}\right\}=\delta_{k m}, \quad\left\{p_{k}, p_{m}\right\}=0, \quad\left\{q_{k}, q_{m}\right\}=0 .
$$

Thus $\left\{p_{k}, q_{k}, k=1,2, \ldots, K\right\}$ can be regarded as Darboux coordinates.

\section{Concluding Remarks}

The peakon sector of the modified Camassa-Holm equation $m_{t}+\left(\left(u_{x}^{2}-u^{2}\right) m\right)_{x}=0$ is not uniquely defined. One way of defining it proposed in $[9,18]$ has a feature that even though the Sobolev $H^{1}$ norm $\|u\|_{H^{1}}^{2}$ is one of the Hamiltonians of the bi-Hamiltonian formulation of this equation in the smooth sector its peakon counterpart does not have this property. In [2] we proposed a different definition of $\mathrm{mCH}$ peakons, based on a different regularization of the ill-defined term $u_{x}^{2} m$. For these (conservative) peakons the $H^{1}$ norm is preserved . In this paper we amplify this statement in the following way:

(1) we introduce a Poisson structure relative to which the conservative peakon equations are Hamiltonian with the Hamiltonian being the very norm $\|u\|_{H^{1}}^{2}$;

(2) using the inverse spectral solutions to conservative peakons put forward in [3] we show the Liouville integrability of the conservative peakon system.

We conclude this Letter by emphasizing that (1.1) is the first equation known to us which has two, natural, peakon sectors. It remains an open question if there are other peakon equations exhibiting this property and, ultimately, what purpose, mathematical or physical, the very existence of these two types of singular solitons imparts to the subject.

\section{Acknowledgements}

The authors thank Stephen Anco for his interest in this work. The Poisson structure used in this Letter has been independently derived by him and announced in his talk during the conference “Nonlinear Evolution Equations and Wave Phenomena", Athens, GA, March 2017. The authors thank Yue Liu for an interesting discussion regarding the dissipative peakons. The authors also thank the referee for critical remarks and for bringing references $[5,6]$ to their attention. Our comments 
in the introduction on the role of the reciprocal transformation are partially in response to his/her suggestions.

Xiangke Chang was supported in part by the Natural Sciences and Engineering Research Council of Canada (NSERC), the Department of Mathematics and Statistics of the University of Saskatchewan, PIMS postdoctoral fellowship and the Institute of Computational Mathematics, AMSS, CAS. Jacek Szmigielski was supported in part by NSERC \#163953.

\section{References}

[1] R. Camassa and D. D. Holm. An integrable shallow water equation with peaked solitons. Phys. Rev. Lett., 71(11):1661-1664, 1993.

[2] X. Chang and J. Szmigielski. Lax Integrability of the Modified Camassa-Holm Equation and the Concept of Peakons. J. Nonlinear Math. Phys., 23(4):563-572, 2016.

[3] X. Chang and J. Szmigielski. Lax integrability and the peakon problem for the modified Camassa-Holm equation. ArXiv e-prints: 1705.06451, 2017.

[4] R. Courant and D. Hilbert. Methods of mathematical physics. Vol. I. Interscience Publishers, Inc., New York, N.Y., 1953.

[5] P. G. Estévez. Generalized Qiao hierarchy in $2+1$ dimensions: reciprocal transformations, spectral problem and non-isospectrality. Phys. Lett. A, 375(3):537-540, 2011.

[6] P. G. Estévez and C. Sardón. Miura-reciprocal transformations for non-isospectral Camassa-Holm hierarchies in $2+1$ dimensions. J. Nonlinear Math. Phys., 20(4):552-564, 2013.

[7] A. Fokas. The Korteweg-de Vries equation and beyond. Acta Appl. Math., 39(1-3):295-305, 1995.

[8] B. Fuchssteiner. Some tricks from the symmetry-toolbox for nonlinear equations: generalizations of the Camassa-Holm equation. Physica D: Nonlinear Phenomena, 95(3):229-243, 1996.

[9] G. Gui, Y. Liu, P. Olver, and C. Qu. Wave-breaking and peakons for a modified Camassa-Holm equation. Commun. Math. Phys., 319(3):731-759, 2013.

[10] A. N. W. Hone and J. P. Wang. Prolongation algebras and Hamiltonian operators for peakon equations. Inverse Problems, 19(1):129-145, 2003.

[11] A. N. W. Hone and J. P. Wang. Integrable peakon equations with cubic nonlinearity. Journal of Physics A: Mathematical and Theoretical, 41(37):372002, 2008.

[12] C. Laurent-Gengoux, A. Pichereau, and P. Vanhaecke. Poisson structures, volume 347 of Grundlehren der Mathematischen Wissenschaften [Fundamental Principles of Mathematical Sciences]. Springer, Heidelberg, 2013.

[13] Y. Matsuno. Bäcklund transformation and smooth multisoliton solutions for a modified Camassa-Holm equation with cubic nonlinearity. J. Math. Phys., 54(5):051504, 14, 2013.

[14] J. Moser. Finitely many mass points on the line under the influence of an exponential potential-an integrable system. pages 467-497. Lecture Notes in Phys., Vol. 38, 1975.

[15] P. Olver and P. Rosenau. Tri-Hamiltonian duality between solitons and solitary-wave solutions having compact support. Phys. Rev. E, 53(2):1900, 1996.

[16] Z. Qiao. A new integrable equation with cuspons and W/M-shape-peaks solitons. J. Math. Phys., 47(11):112701-112900, 2006.

[17] Z. Qiao. New integrable hierarchy, its parametric solutions, cuspons, one-peak solitons, and M/W-shape peak solitons. J. Math. Phys., 48(8):082701, 2007.

[18] Z. Qiao and B. Xia. Integrable peakon systems with weak kink and kink-peakon interactional solutions. Front. Math. China, 8(5):1185-1196, 2013.

[19] O. Ragnisco and M. Bruschi. Peakons, $r$-matrix and Toda lattice. Phys. A, 228(1-4):150-159, 1996. 